\title{
GLA: Gate-Level Abstraction Revisited
}

\author{
Alan Mishchenko Niklas Een Robert Brayton \\ Department of EECS, University of California, Berkeley \\ \{alanmi, brayton\}@eecs.berkeley.edu niklas@een.se
}

\begin{abstract}
Verification benefits from removing logic that is not relevant for a proof. Techniques for doing this are known as localization abstraction. Abstraction is often performed by selecting a subset of gates to be included in the abstracted model; the signals feeding into this subset become unconstrained cut-points. In this paper, we propose several improvements to substantially increase the scalability of automated abstraction. In particular, we show how a better integration between the BMC engine and the SAT solver is achieved, resulting in a new hybrid abstraction engine, that is faster and uses less memory. This engine speeds up computation by constant propagation and circuit-based structural hashing while collecting UNSAT cores for the intermediate proofs in terms of a subset of the original variables. Experimental results show improvements in the abstraction depth and size.
\end{abstract}

\section{Introduction}

Localization abstraction plays an important role in reducing the complexity of formal verification. The known abstraction methods can be classified as follows:

- Automatic vs. manual

- SAT-based vs. BDD-based vs. other

- Proof-based vs. counterexample-based vs. hybrid

- Flop-level vs. gate-level

The first criterion asks whether abstraction is performed automatically by a tool or manually by a verification engineer. The chosen abstraction can be based on different computation methods; SAT-based techniques have largely replaced BDD-based and other methods. In SAT-based methods, abstraction refinement can rely on computing a sequence of UNSAT cores [16] or counterexamples [17] or by applying a hybrid approach, which utilizes both proofs and counterexamples [1][10]. Finally, in terms of the granularity, abstraction can be flop-level [17][10] or gatelevel [3], depending on whether it is constructed using entire next-state logic cones or single gates as primitives.

Using the above taxonomy of abstraction methods, the method presented in this paper can be classified as automatic, SAT-based, hybrid, and gate-level.

The main contribution of this work is in extending the previous hybrid (counterexample-based and proof-based) abstraction [10] in a way that substantially improves scalability of the abstraction engine.

Other contributions include: (a) a new way of computing partial UNSAT cores, resulting in $100 \mathrm{x}$ reduction in

\author{
Jason Baumgartner Hari Mony Pradeep Nalla \\ IBM Systems and Technology Group \\ \{baumgarj, harimony\}@us.ibm.com pranalla@in.ibm.com
}

memory, compared to previous work based on complete proof-logging; (b) a new abstraction refinement framework, which reduces the number of refinement iterations; and (c) a new feature called rollback, which allows the SAT solver to return to a previously marked point in solving a problem.

To understand the importance of the main contribution, we start by introducing hybrid abstraction (HA) presented in [10] and outline the two key insights that led to the gatelevel abstraction (GLA) described in the current paper.

The implementation of HA constructs a localization abstraction by iteratively computing (1) counterexamples produced while the BMC engine is working on a time frame, and (2) UNSAT cores generated when the SAT solver proves the abstracted model to be UNSAT up to this time frame. In the latter case, it is said that the SAT solver proved the abstraction to be precise up to this time frame.

Both counterexamples and proofs are equally important for the efficiency of HA. In particular, the counterexamples indicate locations in the circuit structure where abstraction is not adequately precise. This directs the refinement procedure to aggressively add more logic to the abstracted model. When eventually abstraction becomes precise up to a given time frame, an UNSAT core returned by the SAT solver is used to prune superfluous logic, resulting in a smaller abstraction that is still adequately precise. A major performance drawback of $\mathrm{HA}$ is that the BMC procedure [6] producing counterexamples cannot leverage circuitbased simplifications (such as constant propagation and structural hashing) without jeopardizing the proof-logging required by the SAT solver to generate UNSAT cores. Another important drawback of HA is that it relies on the complete proof whose memory requirements run into gigabytes after several minutes of computation. This makes it necessary to store the proofs on disk, which slows down computation and make implementation cumbersome.

The key insight to overcome these limitations is the following: When a precise abstraction up to a given time frame is found, and the abstraction engine is about to start working on the next time frame, we can choose to always keep the gates added to the abstraction so far, that is, never remove them while possibly adding some new gates to make the abstraction more precise in future time frames. Thus when we analyze the UNSAT core at the end of a time frame we always keep all the gates used at the end of previous timeframes.

Since we committed to keep these gates in the abstraction until the end of the run of the abstraction engine, we can allow circuit-based simplification to be applied to them. For 
example, the constant initial state can propagate and structural hashing can reduce the part of the circuit included in the abstraction. This simplification is crucial for the scalability of the SAT solver working on multiple time frames during BMC. Meanwhile, UNSAT cores can be recorded in terms of only gates added to the abstraction in a particular time frame, since the previously added gates are never removed. Once refinement is completed, a new set of gates is added to the abstraction and kept till the end.

Another key insight allows a dramatic reduction in memory usage for proof-logging: After completing a time frame and performing the refinement, we commit to those gates that have been added to the abstraction so far. As a result, future UNSAT cores, if any, can be expressed in terms of only new gates to be added in the next time frame. This allows recording incremental UNSAT proofs and generating cores in terms of relatively few SAT variables.

Moreover, there is no need to store the structure of the UNSAT proof as done by a typical proof-logger. It is enough to associate each clause with a bit-string encoding new SAT variables using positional notation. This insight leads to a 100x reduction in memory, compared to using full proofs recorded by state-of-the-art proof-loggers, such as the one in MiniSAT. In practice, this means going from $1 \mathrm{~GB}$ to $10 \mathrm{MB}$ on a typical model checking instance that takes about 5 minutes to compute a precise abstraction.

The rest of the paper is organized as follows. Section 2 describes the background. Section 3 describes the algorithm. Section 4 reports experimental results. Section 5 concludes the paper and outlines future work.

\section{Background}

The standard definitions related to Boolean algebra, sequential logic networks, And-Inverter Graphs (AIGs), time frame unfolding, bounded model checking (BMC), SAT solving, counterexample- and proof-based abstraction, etc., are assumed to be well known. Only definitions specific to this paper are given below.

A safety property is represented by a sequential AIG. We call this sequential AIG a miter, even if the problem domain is property checking, not equivalence checking.

An AIG gate refers to any object that is found in the AIG: the constant node, a primary input, a flop, an internal AND node, and a primary output.

An abstraction of depth $\mathrm{K}$ is a subset of gates of the sequential miter, such that if only this subset is included in the bounded unfolding of the miter and the rest are treated as unconstrained primary inputs, the resulting bounded property is "true" in the first $\mathrm{K}$ time-frames of the unfolding. For scalability of subsequent verification, this subset of gates should be as small as possible. Although given the heuristic nature of the abstraction-refinement process, generally the resulting abstraction may include some gates which are unnecessary.

The notions "an abstraction", "an abstraction of the miter" and "an abstracted model", are used interchangeably.
An included (excluded) gate is a gate included (not included) in the abstracted model. In the present work, the constant node and the property output are always included.

An incremental UNSAT core in frame $\mathrm{K}$ is defined as follows: Given is (a) a sequential miter, (b) an abstraction, which is precise up to depth $\mathrm{K}-1(\mathrm{~K}>0)$, and (c) a set S of AIG gates not included in the abstraction. For set $\mathrm{S}$ to be an incremental UNSAT core in frame $\mathrm{K}$, the SAT instance containing the following constraints should be unsatisfiable:

- all the gates included in the abstraction in all $\mathrm{K}$ frames;

- all the gates belonging to $\mathrm{S}$ in all $\mathrm{K}$ frames;

- constraints for the initial state in the starting frame;

- the property output fails in frame $\mathrm{K}$.

In this paper, GLA stands for the proposed method called "gate-level abstraction" and for an abstraction produced by this method. In this sense, a GLA of depth K is a set of AIG gate IDs, which should be included in each of the first $\mathrm{K}$ time frames of the unfolding of the abstracted model to guarantee that the property output does not fail in any of the time-frames from 0 to $\mathrm{K}-1$, inclusive.

\section{Algorithm}

\subsection{The main idea}

The proposed method is motivated by the need to improve scalability of abstraction. It differs from other methods in the way it handles gates included in the abstraction while refining the abstraction in the latest time frame.

The gates included in the abstraction before frame $\mathrm{K}$ can be constant-propagated and structurally-hashed to reduce the size of the SAT problem. When the refinement in frame $\mathrm{K}$ is finished, an incremental UNSAT core can be computed only in terms of the new gates. Once this UNSAT core is available, the set of new gates belonging to the core can be added to the abstraction, resulting in the abstraction of depth K. From now on, all the gates included in this abstraction will be considered "old", while some "new" gates may be added in time frame $\mathrm{K}+1$ if refinement of the abstraction is required in this frame.

The abstraction refinement process terminates upon some configurable scenario: e.g., when a specified number of frames is explored, when a resource limit is reached, or when there was no refinement in several recent timeframes. If a true counterexample is discovered at any time, computation terminates, and the counterexample is returned. If the SAT solver exceeds the allotted resources, the current abstraction is returned.

\subsection{Abstraction framework}

The abstraction framework is described in Figure 3.2.

\subsection{Priority-based abstraction refinement}

This subsection describes our priority-based abstraction refinement (PBAR), which is a special case of counterexample-based abstraction refinement. PBAR is orthogonal to GLA and compatible with other abstraction methods. The pseudo-code is in Figure 3.3. 


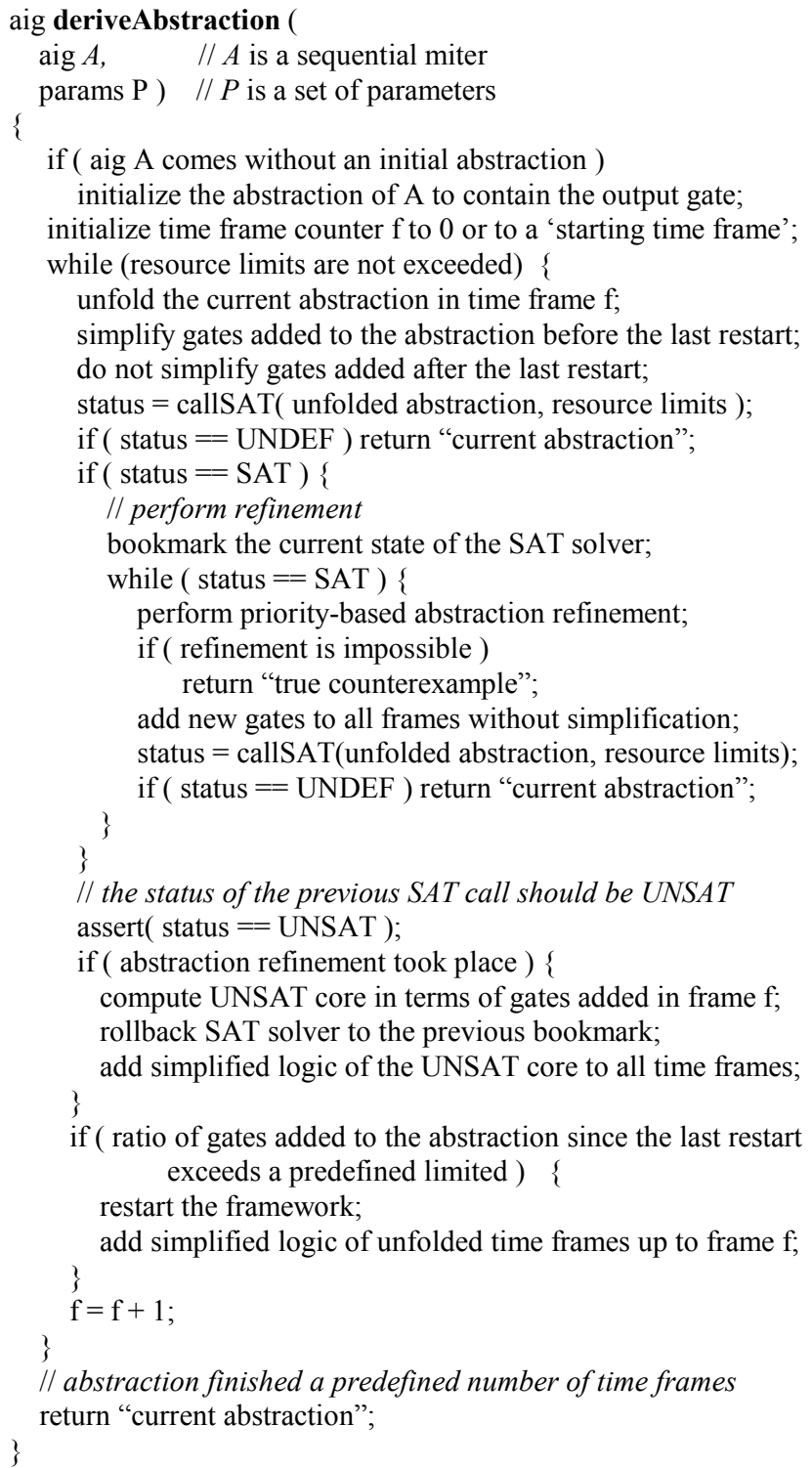

Figure 3.2. Abstraction framework.

Consider the abstracted model, $A^{M}$, and its sequential unfolding for as many time frames as needed to get the property output to fail in the last time frame according to the provided counterexample $\mathrm{C}$. This unfolding is a combinational circuit with a single primary output (PO), which represents the output of the original miter in the last time frame, and two types of primary inputs (PIs), called real PIs (RPIs) and pseudo PIs (PPI), depending on whether they are PIs of the original miter or intermediate cut-points produced by the abstraction engine. Consider also a complete assignment of the PIs, given by the counterexample, which forces the PO to fail.

The refinement algorithm produces a minimal subset of PPIs, such that restricting them to values in the given CEX while keeping other PPIs unconstrained, implies that the property fails. In other words, if it is assumed that these PPIs have these values and the property output does not fail, the resulting SAT problem is UNSAT. If the resulting subset of PPIs is empty, the property failure does not depend on values at the PPIs. It means that the given CEX is a true counterexample to the original verification problem and thus cannot be ruled out by any refinement.

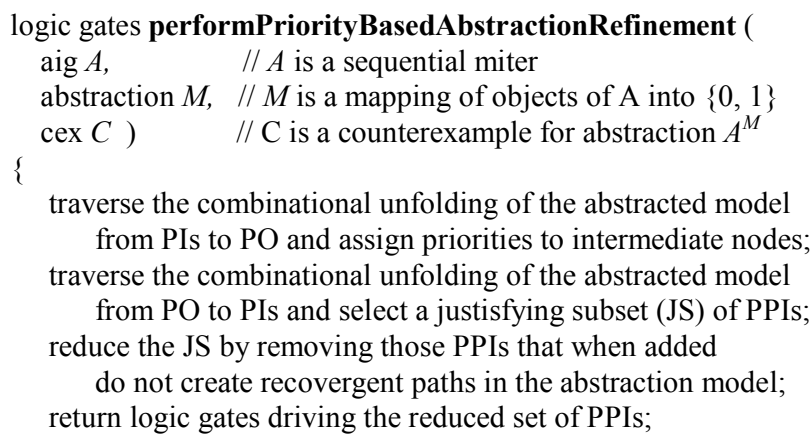

\section{Figure 3.3. Priority-based abstraction refinement.}

Below, such a minimal subset of PPIs is called a justifying subset (JS) of the refinement problem. Note that the JS is not unique. Several JS's of different cardinality can exist for the same refinement problem. However, as our experiments have shown, the number of different JS's in realistic refinement problems is typically not large.

To describe the proposed method for abstraction refinement, it is assumed that an integer number called priority is assigned to each PI. This number indicates how desirable it is to include a PPI into the resulting JS.The assignment of priorities to the PPIs imposes an ordering on them, such that a PPI is only included into the JS if a JS with only PPIs of higher priority does not exist.

When assigning priorities to the PIs, the constant node, the constants representing initial values of the flops in the starting time frame, and the RPIs get the highest priority. This is because we try to use them whenever possible to imply the property output to fail, before including other PPIs into the resulting JS. The PPIs get priority in their natural order of appearance in the topologically traversed AIG of the sequential abstraction model. The PPIs appearing in different time frames get the same priorities.

Assuming that the PPI priority is assigned and each node included in the abstraction is associated in each timeframe with the value it has in the counterexample, a JS of PPIs is constructed in two traversals of the unfolding.

In the first traversal, priority is propagated from PIs to the PO in a topological order. When considering AND nodes, the following rules are used to determine priority:

- If both fanins have counterexample value 1 , the node's priority is the minimum of the priorities of its fanins.

- If both fanins have counterexample value 0 , the node's priority is the maximum of the priorities of its fanins.

- If fanins have different values, the node's priority is equal to the priority of the node fanin whose value is 0 .

At the end of this traversal, each gate of the combinational unfolding has a priority assigned. This 
priority is equal to the lowest priority of a PPI needed to produce the assigned value at a gate. Incidentally, if the priority of the PO is equal to the higher priority assigned to the terminal gates (constants, flop outputs of the starting frames, or RPIs), the PO failure is produced without PPIs, which means a true counterexample has been found.

In the second traversal, the JS is determined by traversing the circuit from the PO to the PIs in a reverse topological order. The following rules are used at each AND node:

- If both fanins have value 1 , both of them are traversed.

- If both fanins have value 0 , only the fanin with a higher priority is traversed.

- If fanins' values differ, only the 0-fanin is traversed.

- If a terminal node (a PI, a constant, or a flop output of the first frame) is reached and the terminal is a PPI, it is added to the JS under construction.

- Immediately after traversing an intermediate node or adding a new PPI to the JS, fanouts of the node or the PPI are traversed and all the nodes whose values are implied by the traversed nodes are labeled as traversed. This allows us to reduce the size of the JS by reusing as many justified intermediate assignments as possible.

The motivation for this is to follow the paths with the highest priority forcing the PO to fail, and to collect the PPIs forming the required JS at the end of each path.

This method works well in practice. It finds small subsets of PPIs useful to refine the GLA while its runtime typically does not exceed $5 \%$ of that of the abstraction engine.

\subsection{SAT solver rollback}

This subsection describes a new feature added to the SAT solver in ABC to enable efficient implementation of GLA.

The SAT solver in $\mathrm{ABC}$ is derived from the original C-version of MiniSAT-1.14 produced by Niklas Sörensson "for those who despise C++" [11]. Since its inclusion in $\mathrm{ABC}$, this version of MiniSAT sustained numerous changes and upgrades, including but not limited to:

- Back-porting of the novel features of MiniSAT 2.0 (e.g. Luby restarts and variable polarity recording).

- Adding the analyze final method used in PDR [12].

- Replacing floating-point-based by integer-based variable activity counting to produce bit-accurate results on different platforms.

- Implementing in-memory proof logging complete with support for UNSAT core computation, interpolation, garbage collection, and proof consistency checking.

- Upgrading the clause database to use memory pages.

The most recently added SAT solver feature motivated by GLA is called rollback. This feature allows the solver to bookmark a state of the clause database. Variables and clauses added by the user or derived by incremental SAT runs can be subsequently removed from the solver by rolling back to the last bookmark. Only one bookmark is currently supported. No attempt is currently made to retain the newly learned clauses, even if they do not depend on the clauses added since the last bookmark.
The rollback mechanism is useful for performing an exploration of the problem space, by bookmarking a state of the solver, adding more clauses, learning something in the process, and later rolling back to the bookmarked point and proceeding in a more desirable direction.

In the context of GLA, bookmarking is used to remember the state of the solver before exploring a new frame. When the exploration is over, possibly after several iterations of refinement, a new UNSAT core is derived. At this point, the rollback removes the logic accumulated in the SAT solver during the refinement. After the rollback, only gates in the last UNSAT core are added to the SAT solver in all timeframes. This guarantees that the unfolding is UNSAT in the last frame while keeping the SAT instance compact for the future computations.

\subsection{Usage details}

This section lists important details related to the implementation of GLA in ABC as command \& $g l a$.

Out of the box, \&gla runs without resource limits. It can be controlled by limiting: runtime $(-\mathrm{T}<$ num $>)$, the $\max$ number of frames covered $(-\mathrm{F}<$ num $>)$, the max conflicts per SAT call $(-\mathrm{C}<$ num $>)$, or the abstraction size $(-\mathrm{R}$ $<$ num $>$ ). With the latter resource limit, \&gla stops when less than a given number of gates is abstracted away.

To view the parameters of the abstraction derived by \&gla, run \&ps. To derive the abstracted model, run \&gla_derive, followed by a call to a prover of your choice.

In the verbose mode $(\& g l a-v)$, the following information is printed while abstraction is being performed:

Column 1: The number of a time-frame.

Column 2: Percentage of objects included in the abstraction.

Column 3: The size of the abstraction in terms of gates (logic nodes and flops, excluding primary inputs).

Column 4: The number of pseudo-primary inputs (PPIs).

Column 5: The number of flip flops (FF).

Column 6: The number of logic nodes (LUT).

Column 7: The number of conflicts in this frame (Confl).

Column 8: The number of CEXes in this frame (CEX).

Column 9: The number of variables in the SAT solver (Vars).

Column 10: The number of problem clauses (Clas).

Column 11: The number of learned clauses (Lrns).

Column 12: Runtime since the beginning (Time).

Column 13: Memory used by SAT solver and proof (Mem).

In summary, the command line to run GLA may look as follows: \& $r<$ file> aig; \&gla -v -T 100; \&gla_derive; \&ps; $\& p u t ; p d r$, where \&gla_derive derives the abstracted model and command $p d r$ tries to prove the abstracted model UNSAT using PDR [12]. Command $\& p s$ prints the ratios of flops/nodes included in the resulting abstraction. Alternatively, command line \& $r$ <ile> aig; \&gla - vq runs abstraction and PDR for the abstracted model concurrently.

Two granularities of CNF construction are supported in \&gla (with switch $-c$ ): logic nodes with up to 5 fanins [12] (default) and 2-input AND nodes. In practice, the former is better because the CNF is more compact (SAT solving is faster) and the granularity of abstraction is increased (resulting in fewer refinement iterations). 


\subsection{Comparison with previous work}

The main difference of GLA compared to previous work on hybrid abstraction [3][10], is the ability to simplify the abstracted part of the circuit and to perform UNSAT core computation incrementally. Minor differences are: reference [3] uses min-cut heuristics while we found them not useful when relying upon PDR [12] as a subsequent proof technique; reference [10] uses flop-based abstraction while we found that a more fine-grain GLA scales better.

A different way of improving the UNSAT core computation during abstraction was developed in [2].

\section{Experimental results}

The proposed approach to abstraction (GLA) is implemented in ABC [5] as command \&gla. GLA is compared against hybrid flop-based abstraction (ABS) [10] and two versions of GLA: GLAnp with UNSAT core based on full proof and GLAn based on incremental UNSAT core. Unlike GLA, both disable constant propagation and structural hashing across the time frames.

The suite of IBM benchmarks from [8] (except two easy SAT instances $6 s 40 p 1$ and $6 s 40 p 2$ are removed) was used in this experiment. The experiment used one core of an Intel Xeon CPU E5-2670 2.60GHz. Learned clause removal, abstraction manager restarts, and early termination, are disabled to reduce "noise", resulting in the following call: \&gla $[-n][-p]-L 0-P 0-R 0-T 300$.

Table 1 shows the initial statistics (name, primary input count, flop count, and AND gate count) and compares the four abstraction engines (ABS, GLAnp, GLAn, and GLA) in terms of the following metrics:

- Depth (abstraction depth achieved in 300 seconds)

- AND (the number of ANDs in the abstracted model)

- Mem (the amount of RAM used for the UNSAT core computation, in megabytes)

At the bottom, "Geom" shows geometric averages of the corresponding columns and "GeomFF" shows geometric averages of flop counts in the abstracted model. (The actual data are omitted due to page limits.) Dashes in the table indicate that the tool core-dumped.

The results lead to the following observations:

- GLA finds abstractions that are 59\% deeper than those found by ABS and 10\% deeper than (GLAn),

- GLA produces abstractions that are close to ABS in terms of flops but $36 \%$ smaller in terms of AND gates,

- GLA and GLAn use on average 500x and 160x less memory for UNSAT cores than GLAnp, which computes a full proof rather than an incremental proof.

\section{Conclusions and future work}

The paper presents a revised approach for SAT-based hybrid gate-level abstraction, which facilitates checking safety properties represented by large sequential miters, when a relatively small abstraction exists. Miters with a few million gates resulting in abstractions with a few thousand gates have been successfully processed by the algorithm.
The method differs from previous work in that it is more scalable (due to constant propagation and structural hashing) and uses less memory (due to incremental UNSAT core computation without complete proof logging).

Experiments show that the improved abstraction engine works well for industrial verification benchmarks, substantially outperforming previous work and comparing favorably against state-of-the-art industrial tools.

Future work may include:

- Improving abstraction refinement by performing a more detailed structural analysis.

- Developing a more scalable way of refining deep failures of abstraction using partial counterexamples.

- Applying a similar approach to make interpolationbased model checking more scalable.

\section{Acknowledgements}

This work is supported in part by SRC contract 1875.001 and NSA grant "Enhanced equivalence checking in cryptoanalytic applications". We also thank industrial sponsors of BVSRC: Altera, Atrenta, Cadence, Calypto, IBM, Intel, Jasper, Mentor Graphics, Microsemi, Real Intent, Synopsys, Tabula, and Verific for their continued support.

\section{References}

[1] N. Amla and K. McMillan. "A hybrid of counterexample-based and proof-based abstraction”, Proc. FMCAD'04, pp. 181-188.

[2] R. Armoni, L. Fix, R. Fraer, T. Heyman, M. Y. Vardi, Y. Vizel, and Y. Zbar, "Deeper bound in BMC by combining constant propagation and abstraction". Proc. ASP-DAC'07, pp. 304-309.

[3] J. Baumgartner and H. Mony, "Maximal input reduction of sequential netlists via synergistic reparameterization and localization strategies". Proc. CHARME'05, pp. 222-237.

[4] J. Baumgartner, H. Mony, V. Paruthi, R. Kanzelman and G. Janssen, "Scalable sequential equivalence checking across arbitrary design transformations", Proc. ICCD'07, pp. 259-266.

[5] Berkeley Logic Synthesis and Verification Group. ABC: A System for Sequential Synthesis and Verification. http://wwwcad.eecs.berkeley.edu/ alanmi/abc

[6] A. Biere, A. Cimatti, E. Clarke, and Y. Zhu. "Symbolic model checking without BDDs". Proc. TACAS'99, pp. 193-207.

[7] A. Biere, AIGER format. http://fmv.jku.at/aiger/

[8] A. Biere, Hardware Model Checking Competition 2011. http://fmv.jku.at/hwmcc11/

[9] R. Brayton and A. Mishchenko, "ABC: An academic industrialstrength verification tool", Proc. CAV'10, LNCS 6174, pp. 24-40.

[10] N. Een, A. Mishchenko, and N. Amla, "A single-instance incremental SAT formulation of proof- and counterexample-based abstraction", Proc. FMCAD'10.

[11] N. Een and N. Sörensson. MiniSAT. http://minisat.se/MiniSat.html

[12] N. Een, A. Mishchenko, and N. Sorensson, "Applying logic synthesis to speedup SAT", Proc. SAT '07, pp. 272-286.

[13] N. Een, A. Mishchenko and R. Brayton, "Efficient implementation of property-directed reachability", Proc. FMCAD'll.

[14] A. Gupta, M. K. Ganai, Z. Yang, and P. Ashar, "Iterative abstraction using SAT-based BMC with proof analysis”. Proc. ICCAD'03.

[15] A. Gupta, M. K. Ganai, and P. Ashar, "Lazy constraints and SAT heuristics for proof-based abstraction'. Proc. VLSI'05, pp. 183-188.

[16] K. McMillan and N. Amla. "Automatic abstraction without counterexamples”. Proc. TACAS'03, pp. 2-17.

[17] D. Wang, P.-H. Ho, J. Long, J. H. Kukula, Y. Zhu, H.-K. Tony Ma, R. F. Damiano, "Formal property verification by abstraction refinement with formal, simulation and hybrid engines". Proc. $D A C^{\prime} 01$, pp. $35-40$ 
Table 1: Experimental evaluation of abstraction engines using IBM HWMCC benchmarks.

\begin{tabular}{|c|c|c|c|c|c|c|c|c|c|c|c|c|c|c|}
\hline Example & PI & $\mathrm{FF}$ & AND & Depth & Depth & Depth & Depth & AND & AND & AND & AND & Mem & Mem & Mem \\
\hline name & Base & Base & Base & $\mathrm{ABS}$ & GLAnp & GLAn & GLA & $\mathrm{ABS}$ & GLAnp & GLAn & GLA & GLAnp & GLAn & GLA \\
\hline $6 \mathrm{~s} 0$ & 207 & 157 & 3549 & 20 & 25 & 24 & 25 & 3522 & 2792 & 2792 & 2749 & 281 & 4 & 1 \\
\hline $6 \mathrm{~s} 1$ & 45 & 291 & 3023 & 9 & 10 & 10 & 10 & 1984 & 1771 & 1771 & 1573 & 473 & 2 & 1 \\
\hline $6 \mathrm{~s} 2$ & 856 & 781 & 11945 & 51 & 194 & 192 & 347 & 9152 & 2647 & 2647 & 1652 & 561 & 4 & 1 \\
\hline $6 \mathrm{~s} 3$ & 156 & 68 & 3504 & 59 & 1312 & 1267 & 1718 & 3480 & 792 & 792 & 745 & 849 & 1 & 1 \\
\hline $6 \mathrm{~s} 4$ & 209 & 202 & 2451 & 1075 & 3005 & 3368 & 4978 & 1720 & 535 & 535 & 477 & 873 & 2 & 1 \\
\hline $6 \mathrm{~s} 5$ & 141 & 2519 & 28985 & 5 & 6 & 6 & 5 & 19090 & 12618 & 12618 & 12639 & 625 & 115 & 1 \\
\hline $6 s 6$ & 168 & 429 & 4771 & 19 & 65 & 67 & 118 & 4743 & 4550 & 4550 & 4550 & 1601 & 16 & 1 \\
\hline $6 \mathrm{~s} 7$ & 45 & 504 & 2530 & 28 & 33 & 34 & 35 & 1811 & 1759 & 1759 & 1765 & 1457 & 2 & 1 \\
\hline $6 \mathrm{~s} 8$ & 86 & 396 & 3016 & 31 & 35 & 35 & 52 & 2600 & 2300 & 2300 & 2285 & 737 & 2 & 1 \\
\hline $6 \mathrm{~s} 9$ & 252 & 607 & 15555 & 29 & 460 & 481 & 569 & 10533 & 996 & 996 & 883 & 2865 & 1 & 1 \\
\hline $6 \mathrm{~s} 10$ & 244 & 598 & 15373 & 14 & 13 & 13 & 12 & 15098 & 10701 & 10701 & 10378 & 1105 & 115 & 29 \\
\hline $6 \mathrm{~s} 11$ & 244 & 598 & 15430 & 14 & 14 & 14 & 12 & 15156 & 13127 & 13155 & 10037 & 985 & 104 & 95 \\
\hline $6 \mathrm{~s} 12$ & 245 & 598 & 15439 & 9 & 10 & 10 & 9 & 15103 & 10848 & 11418 & 10003 & 809 & 32 & 1 \\
\hline $6 \mathrm{~s} 13$ & 439 & 811 & 25083 & 5 & 6 & 6 & 5 & 17233 & 11752 & 11810 & 11929 & 793 & 58 & 1 \\
\hline $6 \mathrm{~s} 14$ & 439 & 811 & 24927 & 6 & 7 & 7 & 6 & 16916 & 12981 & 13030 & 12345 & 1249 & 29 & 1 \\
\hline $6 \mathrm{~s} 15$ & 439 & 811 & 24927 & 6 & 7 & 7 & 6 & 16916 & 12981 & 12981 & 12345 & 1233 & 29 & 1 \\
\hline $6 \mathrm{~s} 16$ & 249 & 608 & 14137 & 9 & 10 & 10 & 9 & 12882 & 10001 & 10001 & 9849 & 1081 & 43 & 1 \\
\hline $6 \mathrm{~s} 17$ & 450 & 819 & 22419 & 6 & 7 & 7 & 6 & 15422 & 12299 & 12299 & 11817 & 1401 & 27 & 1 \\
\hline $6 \mathrm{~s} 18$ & 450 & 819 & 22559 & 6 & 6 & 6 & 5 & 15557 & 11642 & 11642 & 11663 & 873 & 22 & 1 \\
\hline $6 \mathrm{~s} 19$ & 266 & 607 & 14308 & 18 & 429 & 460 & 494 & 10296 & 928 & 928 & 835 & 3033 & 1 & 1 \\
\hline $6 \mathrm{~s} 20$ & 49 & 201 & 30251 & 7 & 7 & 7 & 6 & 28787 & 17962 & 17919 & 16469 & 1713 & 39 & 1 \\
\hline $6 \mathrm{~s} 21$ & 155 & 3795 & 20098 & 100 & 137 & 134 & 172 & 1904 & 1744 & 1744 & 1303 & 1369 & 5 & 1 \\
\hline $6 \mathrm{~s} 22$ & 73 & 1126 & 15983 & 30 & 36 & 36 & 28 & 10588 & 4412 & 4412 & 3457 & 1409 & 6 & 1 \\
\hline $6 \mathrm{~s} 23$ & 12 & 10009 & 61603 & 19 & 19 & 19 & 20 & 771 & 686 & 686 & 673 & 641 & 1 & 1 \\
\hline $6 \mathrm{~s} 24$ & 25 & 1456 & 10537 & 19 & 23 & 23 & 20 & 5695 & 3938 & 3938 & 2174 & 441 & 11 & 1 \\
\hline $6 \mathrm{~s} 25$ & 131 & 1718 & 6615 & 22 & 23 & 23 & 22 & 6582 & 6576 & 6576 & 6570 & 977 & 1 & 1 \\
\hline $6 \mathrm{~s} 26$ & 247 & 2654 & 9980 & 1292 & - & 177 & 86 & 1917 & - & 1869 & 3361 & - & 11 & 1 \\
\hline $6 \mathrm{~s} 27$ & 144 & 2707 & 10239 & 50 & 69 & 69 & 57 & 622 & 598 & 598 & 440 & 969 & 1 & 1 \\
\hline $6 \mathrm{~s} 28$ & 4 & 2269 & 9974 & 128 & 129 & 130 & 128 & 3987 & 3683 & 3277 & 2331 & 633 & 1 & 1 \\
\hline $6 \mathrm{~s} 29$ & 4 & 2247 & 9897 & 55 & 62 & 61 & 51 & 5107 & 4500 & 5046 & 3307 & 1897 & 46 & 1 \\
\hline $6 \mathrm{~s} 30$ & 32994 & 1195 & 102535 & 51 & 61 & 62 & 66 & 1590 & 810 & 810 & 772 & 1225 & 7 & 1 \\
\hline $6 \mathrm{~s} 31$ & 17 & 197 & 1355 & 29 & 34 & 34 & 40 & 387 & 121 & 121 & 150 & 297 & 1 & 1 \\
\hline $6 \mathrm{~s} 32$ & 8 & 1773 & 7482 & 999 & 2062 & 2062 & 2060 & 62 & 2104 & 2104 & 327 & 537 & 1 & 1 \\
\hline $6 \mathrm{~s} 33$ & 31 & 142 & 954 & 26 & 26 & 26 & 26 & 844 & 810 & 810 & 789 & 825 & 1 & 1 \\
\hline $6 \mathrm{~s} 34$ & 77 & 1564 & 9460 & 39 & 28 & 28 & 59 & 500 & 453 & 453 & 971 & 889 & 1 & 1 \\
\hline $6 \mathrm{~s} 35$ & 77 & 1572 & 9608 & 40 & 33 & 33 & 51 & 1252 & 868 & 868 & 877 & 265 & 2 & 1 \\
\hline $6 \mathrm{~s} 36$ & 74 & 1072 & 7155 & 26 & 28 & 28 & 26 & 688 & 636 & 636 & 611 & 1585 & 1 & 1 \\
\hline $6 \mathrm{~s} 37$ & 42 & 753 & 3707 & 79 & - & 94 & 72 & 3015 & - & 1922 & 1461 & - & 26 & 1 \\
\hline $6 \mathrm{~s} 38$ & 343 & 1931 & 10787 & 13 & 18 & 18 & 18 & 7491 & 5750 & 5750 & 5459 & 625 & 7 & 1 \\
\hline $6 \mathrm{~s} 39$ & 65 & 698 & 6712 & 70 & 64 & 60 & 63 & 6498 & 4348 & 5406 & 4544 & 177 & 12 & 1 \\
\hline $6 \mathrm{~s} 40 \mathrm{p} 0$ & 249 & 5608 & 30430 & 39 & 36 & 37 & 35 & 23776 & 13737 & 13602 & 8152 & 41 & 1 & 1 \\
\hline $6 \mathrm{~s} 41$ & 19 & 959 & 3274 & 65 & 74 & 74 & 72 & 2389 & 1892 & 2620 & 2644 & 769 & 4 & 1 \\
\hline $6 s 42$ & 76 & 1211 & 8345 & 28 & 30 & 31 & 29 & 4847 & 4050 & 4050 & 5188 & 713 & 7 & 1 \\
\hline $6 s 43$ & 30 & 965 & 7408 & 71 & 129 & 133 & 284 & 2349 & 1526 & 1526 & 1940 & 1025 & 2 & 1 \\
\hline $6 s 44$ & 76 & 1211 & 8635 & 28 & 30 & 30 & 29 & 5061 & 4605 & 4605 & 4075 & 1169 & 7 & 4 \\
\hline $6 \mathrm{~s} 45$ & 91 & 651 & 4340 & 98 & 99 & 99 & 98 & 3985 & 3569 & 3569 & 3728 & 1409 & 1 & 1 \\
\hline $6 s 46$ & 91 & 651 & 4208 & 98 & 99 & 99 & 98 & 3944 & 3566 & 3566 & 3622 & 1209 & 1 & 1 \\
\hline $6 \mathrm{~s} 47$ & 34 & 815 & 4101 & 274 & 616 & 624 & 892 & 321 & 334 & 338 & 198 & 1377 & 1 & 1 \\
\hline $6 \mathrm{~s} 48 \mathrm{p} 0$ & 72 & 66 & 795 & 8 & 11 & 11 & 10 & 764 & 568 & 568 & 549 & 97 & 1 & 1 \\
\hline $6 \mathrm{~s} 48 \mathrm{p} 1$ & 72 & 66 & 791 & 7 & 12 & 12 & 10 & 763 & 558 & 558 & 563 & 121 & 1 & 1 \\
\hline $6 s 49$ & 17 & 180 & 1020 & 14 & 15 & 15 & 14 & 1003 & 955 & 955 & 946 & 201 & 1 & 1 \\
\hline $6 \mathrm{~s} 50$ & 1570 & 3107 & 16700 & 77 & 311 & 317 & 654 & 1844 & 801 & 801 & 482 & 1905 & 2 & 1 \\
\hline $6 \mathrm{~s} 51$ & 1570 & 3107 & 16701 & 85 & 290 & 298 & 421 & 1758 & 835 & 835 & 460 & 1657 & 2 & 1 \\
\hline $6 \mathrm{~s} 52$ & 35 & 208 & 1228 & 567 & 549 & 549 & 541 & 1074 & 886 & 886 & 910 & 729 & 2 & 1 \\
\hline $6 \mathrm{~s} 53$ & 35 & 208 & 1230 & 536 & 537 & 537 & 536 & 1187 & 1179 & 1179 & 1156 & 385 & 1 & 1 \\
\hline $6 \mathrm{~s} 54$ & 144 & 1660 & 13411 & 46 & 47 & 45 & 38 & 8816 & 7721 & 7721 & 8230 & 1417 & 25 & 1 \\
\hline Geom & & & & 1.000 & 1.486 & 1.493 & 1.585 & 1.000 & 0.716 & 0.724 & 0.638 & 1.000 & 0.006 & 0.002 \\
\hline GeomFF & & & & & & & & 1.000 & 1.074 & 1.082 & 0.990 & & & \\
\hline
\end{tabular}

